\title{
Early-onset colorectal cancer: when hoof beats are zebras
}

\author{
Rebecca L Siegel*,1 (iD \\ ${ }^{1}$ Surveillance \& Health Services Research, American Cancer Society, Atlanta, GA 30303, USA \\ *Author for correspondence: Tel.: +1 404329 7992; Rebecca.siegel@cancer.org
}

\section{"The stark and rising volume of cases and deaths under 50 is a call to action."}

First draft submitted: 16 April 2020; Accepted for publication: 1 May 2020; Published online: 19 October 2020

Keywords: delays in diagnosis $\bullet$ early-detection $\bullet$ early diagnosis $\bullet$ opportunities $\bullet$ screening $\bullet$ treatment side-effects - young-onset colorectal cancer

By all accounts, colorectal cancer (CRC) was a success story in the USA, one of the few countries where overall incidence and mortality have dropped precipitously because of a combination of changes in risk factors, increased screening and advances in treatment. However, these trends mask a completely different scenario playing out among young generations. For reasons that remain unknown, CRC incidence has increased steadily in Generation 'X'ers and Millenials since at least the mid-1990s, with mortality following suit a decade later [1,2]. As Doll taught years ago, trends in young adults are 'by far the most important for assessing our progress against cancer' because they reflect relatively recent carcinogenic exposures as opposed to those accumulated over a lifetime [3]. According to a recent analysis reflecting $95 \%$ of the United States population, CRC incidence in people younger than 50 years, referred to as early-onset CRC (EOCRC), is rising by approximately $2 \%$ per year for both colon and rectal tumors and rates have also begun increasing in individuals aged 50-64 [4]. This trend, in parallel with continued declines in older individuals, has shifted the median age of CRC diagnosis from 72 years to 66 years, in little more than a decade. In 2020, approximately 18,000 people in their 20 's, 30's, or 40's will hear the words 'you have colorectal cancer', the equivalent of 49 new cases per day [4]. These individuals are more likely than their older aged counterparts to be diagnosed with metastatic disease; experience long-lasting side effects of treatment, such as infertility; and to suffer financial and psychosocial aftershocks of treatment. Further, three in four EOCRC patients are caring for children younger than 18 years at the time of diagnosis [5]. Although it will probably be years before research uncovers the early-life exposures that are elevating risk, there are numerous opportunities now to reduce the burden for these patients.

EOCRC patients are diagnosed with advanced disease $40 \%$ more often than their older counterparts - even after accounting for screening [6], largely because of avoidable delays in diagnosis. Studies indicate a total average time to diagnosis of 217-271 days, with the interval from symptom onset to first medical contact being the largest contributor to age-related disparities. Young patients have experienced symptoms for twice as long as older individuals at initial presentation, postponing care for a variety of reasons including embarrassment; competing demands on time related to work and family; and lack of access to healthcare. Those younger than 50 are threetimes more likely to be uninsured than older individuals, and even with insurance they are less likely to have a usual source of care or utilize primary care, especially men. Delays also occur because of misdiagnosis with more common conditions, such as hemorrhoids in patients who present with rectal bleeding. The American Society for Gastrointestinal Endoscopy (IL, USA) recommends endoscopic evaluation of all patients with lower gastrointestinal bleeding, regardless of age. Earlier diagnosis could also be facilitated through educational campaigns to destigmatize the disease, increase awareness of symptoms and inform primary care clinicians about elevated risk in younger cohorts. Closing gaps in access to care is more challenging but could have an even larger impact on outcomes. A recent analysis demonstrated that implementation of the Affordable Care Act provision that expanded dependent insurance coverage to age 26 was associated with both earlier stage diagnosis and more timely treatment among CRC patients aged 19-25 years [7]. Even a small shift could translate into many lives saved; almost $80 \%$ of EOCRC 
patients diagnosed with regional spread survive five years compared with only $20 \%$ diagnosed with metastatic disease [4]. Moreover, a recent study estimated that almost $40 \%$ of all CRC deaths could be averted if patients who were diagnosed with stage IV disease were instead diagnosed at stage III [8].

EOCRC could also be diagnosed earlier and in some cases prevented altogether if more people were screened according to their disease risk. Up to one-third of EOCRC patients have a genetic predisposition or family history of the disease. Individuals with a first-degree relative diagnosed with CRC or advanced adenoma are two to four-times more likely to develop the disease than people without this family history and are recommended to begin screening by age 40 . Yet, among adults who have this family history, $60 \%$ of those in their 40 's have not been screened [9], and more than $60 \%$ of those younger than 50 have never been asked for this information by their physician [10]. The single most important predictor of screening adherence is a physician recommendation. Better attention to taking and regularly updating family history and acting on that information would identify young adults at high risk and reduce the incidence of advanced disease. A recent study found that among EOCRC patients in their 40's who met criteria for early screening, 98\% would have been diagnosed earlier and some cases possibly prevented, if they had been screened according to guidelines [11]. Those at even higher risk because of a hereditary syndrome should begin screening in their 20 's, but most are unrecognized until their cancer diagnosis, including the 1.2 million Americans with Lynch syndrome. Earlier identification and surveillance of individuals with elevated hereditary risk is both cost-effective and lifesaving. However, there is little movement on early screening associated with familial risk and only $15 \%$ of community hospitals have integrated recommended universal testing of EOCRC tumors for Lynch syndrome [12]. Hopefully the model put forth by Cohen and colleagues for successful implementation of Lynch syndrome screening will not only increase adoption, but also facilitate more rapid dissemination of emerging genomic technologies [13], including multigene panel testing, which was recently recommended for all EOCRC by the Collaborative Group of the Americas on Inherited Gastrointestinal Cancer to benefit the large number of high-risk families with other germline mutations [14].

CRC screening for those without familial or other risk factors (for example, inflammatory bowel disease) should begin at age 45 according to recent (American Cancer Society; GA, USA) guidelines, but at age 50 according to other organizations whose guidelines were issued prior to newer evidence on the epidemiology of EOCRC. Many of these organizations recommend screening at 45 for African Americans, who are at elevated risk overall, but have rates of EOCRC now comparable to those in the white/Caucasian demographic- who have experienced the steepest increase [4]. Screening at 45 could potentially detect cancer earlier for some EOCRC patients, given that almost half are 45-49 years at diagnosis, although older adults will reap most of the benefit of initiation at 45 versus 50 . The new American Cancer Society guidelines have received substantial criticism, partly based on lack of empirical evidence to support screening efficacy in ages 45-49. However, clinical and molecular features of CRC are similar among individuals in their 40's and 50's [15], and it is doubtful that screening in this age group would be any less effective at detecting sporadic cancers than at detecting common familial cancers or those in African Americans, for which there is widespread agreement for screening before 50 .

The burden of EOCRC could also be lessened through approaches to treatment and patient support that are more focused on the unique but universal needs of these patients. Young survivors report that the largest gap in their care is lack of basic information about the physical, emotional and financial toll of diagnosis and treatment. For example, although EOCRC patients are in their peak sexual and childbearing years, only a small fraction of physicians initiate conversations regarding the impact of treatment on sexuality and reproductive health. This information is especially critical for the $40 \%$ of EOCRC patients with rectal tumors, who are at a higher risk of sexual dysfunction, body image distress and infertility. Surveys indicate that most EOCRC survivors were either unaware that sexual dysfunction and infertility were a potential consequence of treatment or were too embarrassed to ask. 'Do no harm' extends beyond providing appropriate treatment. Clinicians have an obligation to initiate discussions about treatment side effects and empower young patients to ask questions throughout their cancer experience. The provision of information and resources about pretreatment options for fertility preservation and interventions for sexual dysfunction should be standard practice. Improved management of expectations and support for bowel function, cognitive deficits and challenges with body image are also needed.

EOCRC patients are also ill-equipped to manage the profound economic burden that accompanies diagnosis. Compared with older patients, younger adults are less financially secure and more likely to suffer lost earnings, career disruptions and bankruptcy as a result of their diagnosis. Physical side effects of treatment often reduce work performance and productivity, increasing risk for job loss. Financial stress among young patients is associated with reduced treatment compliance and lower quality of life, and is exacerbated by the rising cost of CRC therapeutics. 
Over treatment among young patients is also a growing concern. After controlling for tumor characteristics, colon cancer patients younger than 50 are more likely than older adults to receive adjuvant chemotherapy and are twice as likely to receive multi-agent regimens despite marginal survival benefit [16]. Multi-agent CRC therapies are associated with persistent long-term toxicities and higher expense. Further, half of young patients receive chemotherapy for low-risk stage II disease although it is not standard of care and has no impact on survival. Providers can play a large role in improving quality of life and mitigating financial hardship by simply sharing more information about costs and side effects and collaboratively designing a treatment plan that considers patient needs and preferences. Providing additional resources, such as patient assistance programs, can also help mitigate financial toxicity.

The face of CRC has changed rapidly in the nearly two decades since the first population-based report of rising incidence in young adults, with the disease now striking our children and grandchildren, as well as our parents and grandparents. The stark and rising volume of cases and deaths under 50 is a call to action. While much important research on the etiology of EOCRC has begun, little attention is focused on easing the burden for patients of today. Simple common-sense interventions would improve outcomes and raise quality of life across the cancer continuum. Late stage diagnosis is a hallmark of EOCRC that could be attenuated through more risk-based screening and targeted educational campaigns that lift the stigma from the disease and its symptoms. History demonstrates that increasing awareness stimulates action. Katie Couric's brief informational segment during one week of a morning television program in 2000 spiked screening rates for nine months and president Reagan's diagnosis prompted a similar response [17]. The EOCRC experience could also be eased through more holistic treatment and survivorship management, which includes comprehensive information and communication regarding patient reproductive, sexual, psychosocial and economic health. Connecting patients with support groups, such as COLONTOWN, and resources for financial assistance and social and mental health is also invaluable. A CRC diagnosis is difficult at any stage of life, but during the bustling years of early adulthood it is terrifying. It is time to unite and provide better care for the new generation of CRC patients.

\section{Financial \& competing interests disclosure}

The author has no relevant affiliations or financial involvement with any organization or entity with a financial interest in or financial conflict with the subject matter or materials discussed in the manuscript. This includes employment, consultancies, honoraria, stock ownership or options, expert testimony, grants or patents received or pending, or royalties.

No writing assistance was utilized in the production of this manuscript.

\section{Open access}

This work is licensed under the Attribution-NonCommercial-NoDerivatives 4.0 Unported License. To view a copy of this license, visit http://creativecommons.org/licenses/by-nc-nd/4.0/

\section{References}

1. Siegel RL, Miller KD, Jemal A. Colorectal cancer mortality rates in adults aged 20 to 54 years in the United States, 1970-2014. JAMA 318(6), 572-574 (2017).

2. Siegel RL, Fedewa SA, Anderson WF et al. Colorectal cancer incidence patterns in the United States, 1974-2013. J. Natl. Cancer Inst. 109(8), djw322-djw322 (2017).

3. Doll R. Progress against cancer: an epidemiologic assessment. The 1991 John C. Cassel Memorial Lecture. Am. J. Epidemiol. 134(7), 675-688 (1991).

4. Siegel RL, Miller KD, Goding Sauer A et al. Colorectal cancer statistics, 2020. CA Cancer J. Clin.70(3), 145-164 (2020).

5. Colorectal Cancer Alliance. Young-onset colorectal cancer survey report. (2018). ccalliance.org/about/never-too-young/survey/2018-young-onset-colorectal-cancer-survey-report

6. Chen FW, Sundaram V, Chew TA, Ladabaum U. Advanced-Stage Colorectal Cancer in Persons Younger Than 50 Years Not Associated With Longer Duration of Symptoms or Time to Diagnosis. Clin Gastroenterol Hepatol 15(5), 728-737.e723 (2017).

7. Nogueira L, Chawla N, Han X, Jemal A, Yabroff KR. Colorectal cancer care among young adult patients after the dependent coverage expansion under the affordable care act. J. Natl Cancer Inst. doi:10.1093/jnci/djz235 (2019) (Epub ahead of print).

8. Clarke CA, Hubbell E, Kurian AW, Colditz GA, Hartman A-R, Gomez SL. Projected reductions in absolute cancer-related deaths from diagnosing cancers before metastasis, 2006-2015. J. Cancer Epidemiol. Biomarkers Prev. doi:10.1158/1055-9965.EPI-19-1366 (2020) (Epub ahead of print).

9. Tsai MH, Xirasagar S, Li YJ, De Groen PC. Colonoscopy screening among US adults aged 40 or older with a family history of colorectal cancer. Prev. Chronic Dis. 12, E80 (2015). 
10. Fletcher RH, Lobb R, Bauer MR et al. Screening patients with a family history of colorectal cancer. J.Gen. Intern. Med. 22(4), 508-513 (2007).

11. Gupta S, Bharti B, Ahnen D et al. Potential impact of family history-based screening guidelines on the detection of early-onset colorectal cancer. Cancer doi:10.1002/cncr.32851 (2020) (Epub ahead of print).

12. Beamer LC, Grant ML, Espenschied CR et al. Reflex immunohistochemistry and microsatellite instability testing of colorectal tumors for lynch syndrome among US Cancer programs and follow-up of abnormal results. J. Clin. Oncol. 30(10), 1058-1063 (2012).

13. Cohen SA, Laurino M, Bowen DJ et al. Initiation of universal tumor screening for Lynch syndrome in colorectal cancer patients as a model for the implementation of genetic information into clinical oncology practice. Cancer 122(3), 393-401 (2016).

14. Heald B, Hampel H, Church J et al. Collaborative Group of the Americas on Inherited Gastrointestinal Cancer Position statement on multigene panel testing for patients with colorectal cancer and/or polyposis. Fam. Cancer doi:10.1007/s10689-020-00170-9 (2020) (Epub ahead of print).

15. Willauer AN, Liu Y, Pereira AaL et al. Clinical and molecular characterization of early-onset colorectal cancer. Cancer 125(12), 2002-2010 (2019).

16. Kneuertz PJ, Chang GJ, Hu C-Y et al. Overtreatment of young adults with colon cancer: more intense treatments with unmatched survival gains. JAMA Surg. 150(5), 402-409 (2015).

17. Cram P, Fendrick AM, Inadomi J, Cowen ME, Carpenter D, Vijan S. The impact of a celebrity promotional campaign on the use of colon cancer screening: the katie couric effect. Arch. Intern. Med. 163(13), 1601-1605 (2003). 